\title{
THE RESULTS OF INVESTIGATIONS OF THE PULKOVO HORIZONTAL MERIDIAN CIRCLE (HMC)
}

\author{
G. I. PINIGIN, L. A. SUKHAREV, and G. M. TIMASHKOVA \\ Pulkovo Observatory, U.S.S.R.
}

The observations in R.A. with the Pulkovo HMC and investigations of the instrument, reported in Trans. IAU XIVA (p. 39), are being continued during the past two years (Pinigin, 1972, 1973). Some of the new results are as follows:

(1) The foundation of the HMC was made according to the scheme published by Sukharev (1955). A stability of the north and south collimators is characterized by the azimuth variation of \pm 0 ". 4 , which corresponds in practice to the stability of the meridian marks of the Pulkovo large transit instrument.

(2) The mirror of the HMC is a monolithic steel block with a rotation axis. The temperature deformations of it are negligible. The collimation of the first and second reflecting surfaces of the mirror can be represented by the formulas

$$
\begin{aligned}
C^{\mathrm{l}} & =-0^{\prime \prime} .29+0^{\prime \prime} .0040\left(t^{\circ}+0.7\right)-0^{\prime \prime} .100 \Delta T \\
C^{\mathrm{II}} & =-0^{\prime \prime} .85-0.0045\left(t^{\circ}+0^{\circ} .7\right)+0^{\prime \prime} .060 \Delta T,
\end{aligned}
$$

where $t^{\circ}$ is the temperature in the pavilion, $\Delta T=T-1969.75$ - time in years.

(3) The two coordinate wedge micrometers allow autocollimation measurements to be made by the photoelectric method with an accuracy of \pm 0 " $01-00^{\prime \prime} 02$. This result confirms the good quality of the optical system, which has the following construction:

(a) the objectives of the collimators are composed of two lenses, crown and flint with similar coefficients of linear expansion;

(b) the lenses are put into the self-centering casing of the bearing type. The lenses rest upon the bearings under the action of their weight. The bearings, which are like those of a classical meridian instrument, are fixed on the pillars. The eye-piece systems of the collimators have similar construction. The micrometer tube is fastened in the center of the disc made of crown glass;

(c) the collimator tubes are not connected either with the objectives or the eyepieces or pillars. They serve for protection from outside influences only. Each tube consists of two elements; a massive steel inner tube and a light aluminium outer one. There is a special ventilating system between them.

(4) The pivots of the HMC mirror rest upon box-wood bearings. Such a system of support preserved the figure of pivots during 1.5 years of operating.

(5) Seasonal variations of the instrumental system $\left(\Delta n_{\delta}\right)$ have not been detected in practice within the limits of \pm 0 s 01 .

(6) The mean error of one determination of right ascension is about \pm 0 s $011 \sec \delta$ for upper culmination and $\pm 0.013 \sec \delta$ for lower culmination. 
All these investigations show that the Pulkovo HMC can be used for precise determinations of R.A. At present the HMC is being prepared for observations of declinations.

\section{References}

Pinigin, G. I.: 1972, Trudy 18th Astrometr. Konf., p. 158.

Pinigin, G. I.: 1973, Trudy 19th Astrometr. Konf., in press.

Sukharev, L. A.: 1955, Trudy 12th Astrometr. Konf., p. 189. 